\title{
Detection of renal allograft rejection by computer
}

\author{
IAN M TRIMBLE, MICHAEL WEST, MARTIN S KNAPP, ROY POWNALL, ADRIAN F M SMITH
}

\begin{abstract}
A computer program incorporating an adaptation of a statistical method, the multiprocess Kalman filter, was used to detect changes in trends of plasma creatinine and urea concentrations. In 28 recipients of renal allografts a definite deterioration in renal function was identified retrospectively on 32 occasions by an experienced renal physician independently of the statistical analysis. The computer identified 31 of these 32 episodes using creatinine and urea results, and 29 using creatinine alone. Dysfunction was identified by the computer significantly earlier $(p<0.05)$ than by the clinician and a median of one day earlier $(p<0.02)$ than treatment was actually initiated. The computer identified dysfunction on 11 out of 1259 days when the clinician did not suspect rejection. These 11 episodes may have had a pathological importance, though no clinical diagnosis was made.
\end{abstract}

This computer method is useful for immediate analysis of incoming results and for timing events either prospectively or retrospectively.

\section{Introduction}

Plasma creatinine and urea concentration are the measurements most widely used for detecting renal allograft rejection, with a rise indicating a deterioration in renal function. The results are rarely considered in a way that makes best use of their potential to define events. A review of 26 consecutive papers from

Renal Unit, City Hospital, Nottingham NG5 1PD

IAN M TRIMBLE, BM, MPHIL, medical student MARTIN S KNAPP, MD, FRCP, consultant physician ROY POWNALL, BSC, PHD, senior scientific officer

Department of Mathematics, University of Nottingham, Nottingham ADRIAN F M SMITH, MA, PHD, professor of mathematical statistics MICHAEL WEST, BSC, PHD, postgraduate research student

Correspondence and requests for reprints to: Dr Martin S Knapp, Unit of Medical Information Technology, University of Nottingham, Queen's Medical Centre, Nottingham NG7 2UH. publications concerned with tests investigated for their potential as monitors of human allografts showed a wide range in the incidence of rejection episodes, whether these were considered as episodes per patient $(0 \cdot 16-3 \cdot 86)$ or as episodes per patient month $(0 \cdot 11-2 \cdot 00)$. Objective criteria for rejection were defined as arbitrary rises in serum creatinine concentration in two of the 26 papers and were omitted completely from the remaining 24 . Recently published reports on new immunological methods, ${ }^{12}$ that claim to be able to diagnose rejection earlier also fail to define the onset of the decrease in renal function due to rejection with an adequate analysis of plasma creatinine values.

We have advocated a simple regression method to help define the onset of functional change, using graphs of plasma creatinine concentration transformed to the reciprocal and "corrected" for acute changes in body weight. ${ }^{3}$ These graphs help time the onset of rejection but necessitate a subjective assessment of the information, and a more objective analysis was considered necessary. The Kalman filter ${ }^{4}$ was chosen and adapted to define in quantitative terms any change in trends shown by sequences of the plasma creatinine or urea concentrations. The statistical probability of change could then be used to define objectively when an acute failure of renal function, usually due to rejection, had occurred.

Similar sequences of observations are collected in the course of many clinical and experimental studies, and failure to apply appropriate statistical techniques may result in important facts about change in trend remaining undetected, or being described only subjectively. Changes in the pattern of observed sequences may be masked by "noise" in the system, resulting from biological variability, and from errors in collecting, measuring, and processing of data. Statistical techniques are sometimes applied to the analysis of series of clinical data-for example, simple regression, ${ }^{3}$ stepwise regression analysis, ${ }^{5}$ simple Cusum plus a decision "mask", ${ }^{6}$ two state Kalman filter, ${ }^{7}$ and multistate Cusum and Bayesian forecasting techniques. ${ }^{8} 9$ Other statistical procedures are used by non-biological scientists to detect changes in "noisy" sequences of data, and we chose to adapt a sophisticated technique, the multistate Kalman filter of Harrison and Stevens, ${ }^{4}$ to determine one-step back probabilities of change. We incorporated with it a system that adjusts the sensitivity of the system automatically in response to changes in noise in the test series. ${ }^{10} \mathrm{We}$ outline the principles of the procedure, together with techniques employed to reduce the noise in the series be- 
fore analysis. The techniques also appeared potentially useful for the prospective monitoring of day to day results as they arrive in the course of day to day care of patients and we aimed to adapt this technique for this purpose.

\section{Patients and methods}

Twenty eight consecutive recipients of renal allografts were studied. In all cases grafts functioned sufficiently to lower plasma creatinine. Plasma creatinine and urea concentrations were measured on at least five days of every week for the first 60 days after grafting or until the graft was considered irreversibly rejected. A total of 19 of the 28 grafts were functioning after 60 days. The other nine grafts were rejected; these patients were monitored for a mean of 24 days. Patients were weighed on the days that blood samples were taken.

Morning specimens of venous blood taken between 0900 and 1100 hours were analysed for plasma constituents and the results used for evaluation by clinicians and for the statistical analysis. In some patients more than one sample was taken in one day; these results were available to the clinical teams but were not used in the statistical analysis reported here.

Plasma creatinine and urea concentrations were measured on an instrumentation laboratory 919 analyser, using the Jaffe reaction for creatinine (coefficient of variation $=3.6 \%$ ), and the urease/glutamate dehydrogenase procedure for urea (coefficient of variation $=1.7 \%$ ).

\section{CATEGORIES OF CLINICAL REJECTION}

All the information collected during the 60 days, including flow charts, clinical notes, special investigations (often including graft biopsy), and details of treatment, was summarised and presented to an experienced renal physician (MSK). Plasma creatinine and urea concentrations, corrected for acute changes in hydration and transformed to the reciprocal, were presented as a graphical plot of the full 60 days, but no statistical analyses were available. Given this information, and knowledge of the patient's subsequent clinical course, each day was graded on a seven point scale assessing the likelihood that the patient was undergoing rejection on that day (table I). Using the information provided there were 28 occasions on which a deterioration in renal function was considered by the physician to be due to rejection (category 6 ). This gives an incidence of 1.0 episodes per patient per month, which is within the range of values noted by others.

\section{MATHEMATICAL ANALYSIS OF DATA}

Before applying complex statistical methods it was considered crucial to identify possible sources of noise and to take account of these when developing the monitoring procedure. There are obvious sources of noise, directly connected with variability in laboratory measurements but the following also appeared important: "rounding errors," resulting from the truncation of recorded digits, "timing errors," resulting from observations being assumed to be collected at the "official" rather than the actual time of measurement; and distortions introduced into measurements of some blood constituents by changes in hydration ${ }^{11}$ (fig 1). The reciprocal transformation of plasma creatinine concentrations was used, with renal function manifesting itself as a series of approximately linear trends, whose direction changed as function moved from improvement to deterioration, or vice versa. $^{311}$ The multiprocess Kalman filter model was then developed for a situation with four states (fig 2).101213 In addition, a procedure for learning about unknown error in the system, developed by West and Smith, ${ }^{10}$ was found to be a considerable improvement over the method proposed by Harrison and Stevens ${ }^{4}$ and overcame the criticisms of the Kalman filter made by Stoodley and Mirnia. ${ }^{9}$

To arrive at optimal clinical decisions the calculated probabilities may be combined with specific assessments of utility, such as those adopted by van der Helm and Hische ${ }^{12}$ for considering blood glucose values. The interpretation of utility assessments requires expert subjective opinion, however, so receiver-operator characteristic (ROC) curves were chosen to define an objective cutoff value above which results were deemed to indicate that a significant deterioration in renal function had occurred. ${ }^{13}$

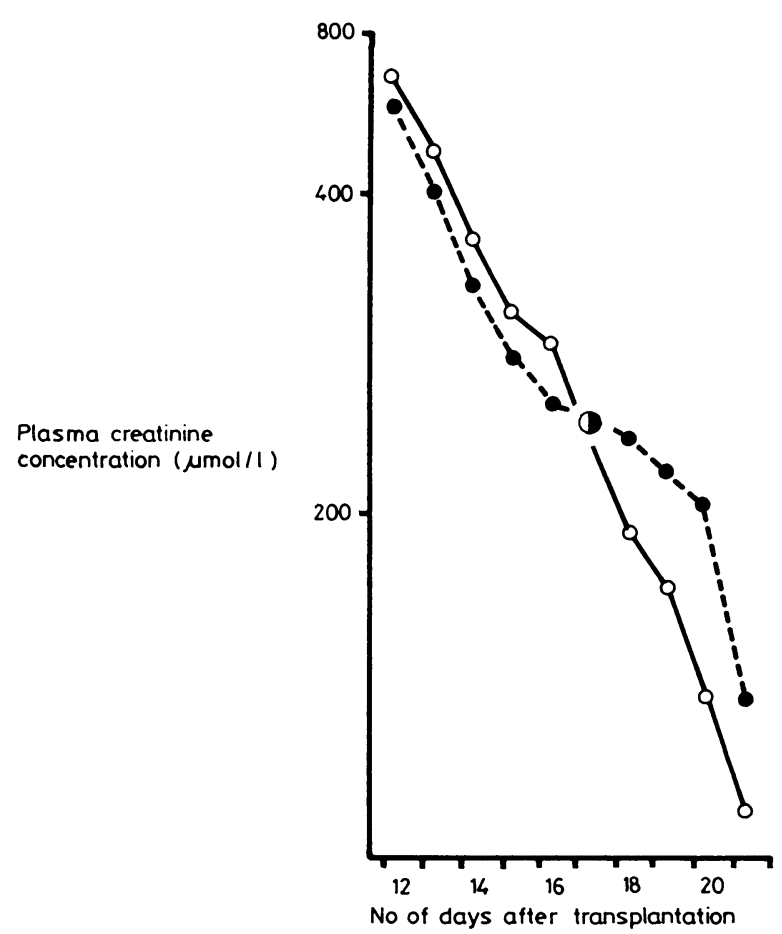

FIG 1-A series of plasma creatinine concentrations (9), when adjusted for changes in weight reflecting alterations in volume of distribution, shows a decrease in amount of "noise" in trend observed $(O) .^{11}$
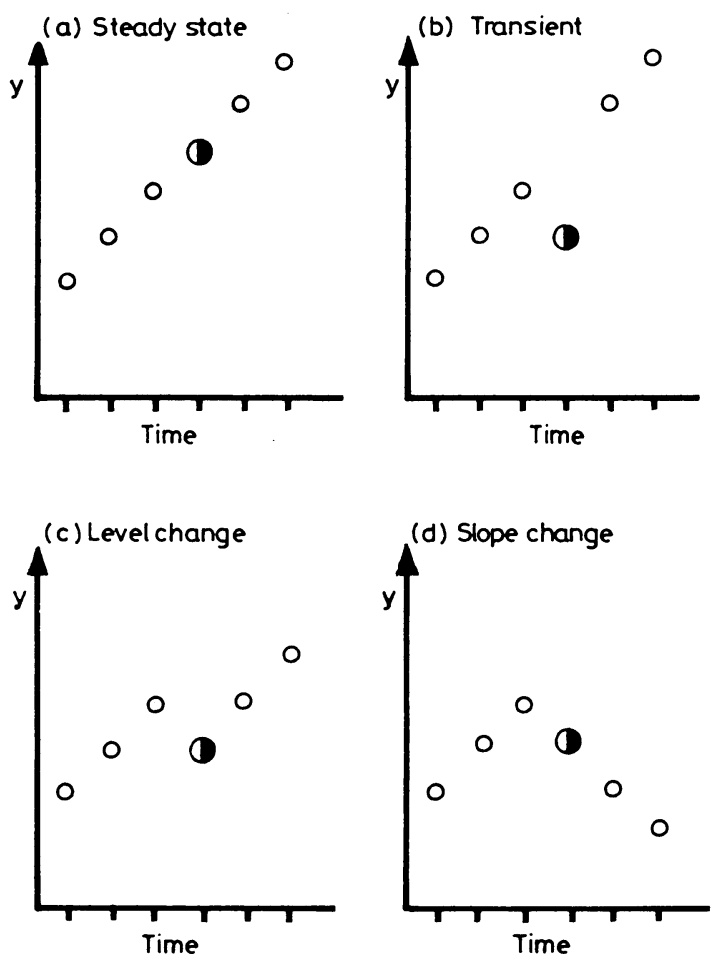

FIG 2-As a new data point ( 0 ) becomes available there are at least four possibilities: (a) system is in a steady state-no abrupt changes have taken place and previous trend continues; (b) point is a transient or outlier-for example, a laboratory error; $(c)$ point corresponds to a sudden change in overall level of sequence-for example, after dialysis; or $(d)$ point corresponds to a sudden change in slope-for example, a deterioration in renal function due to rejection. When the next data point becomes available recursive estimation procedure may be used to determine the probability that a particular change occurred at previous data point. 
Ideally a test should have a high specificity and sensitivity (correspondingly, a low false positive ratio and high true positive ratio). To achieve a high predictive value for the test a high specificity was required and this was achieved by choosing an arbitrary, but constant and reproducible ratio of true positive to false positive of $3: 1$. This gave a cutoff for weight-corrected creatinine of $0 \cdot 195$. Any result exceeding this has a predictive value, calculated from the results in table I, greater than $0 \cdot 72$-that is, given a result exceeding $0 \cdot 195$, the probability that a significant deterioration in renal function has occurred exceeds $0 \cdot 72$. The corresponding predictive values at the cutoff were 0.63 for urea and 0.73 for uncorrected creatinine.

Figure 3 illustrates the application of the techniques to two different series of data; in both examples a significant deterioration in function was detected before the plasma creatinine concentration exceeded $200 \mu \mathrm{mol} / \mathrm{l}(2.3 \mathrm{mg} / 100 \mathrm{ml})$.

\section{DEFINITION OF TIME OF ALLOGRAFT REJECTION}

For clinical rejection category 6, MSK estimated the time he would have started antirejection treatment given the hypothetical assumptions $(a)$ early treatment is beneficial, $(b)$ too much treatment is dangerous, and $(c)$ the patient's state of health at the time would not affect the decision to treat. This defined the time of retrospective clinical diagnosis of rejection. The time of prospective clinical diagnosis was taken as the time when antirejection treatment was actually started; this was the only objective evidence as to the time when the clinical team agreed rejection was occurring. The clinical estimates were made to the nearest half day as they were ocasionally based on the results from evening specimens. The time of computer diagnosis of rejection was defined as the day on which the probability that a slope change had occurred (fig 3) exceeded the cutoff value. This is a prospective diagnosis of rejection as the computer analyses results sequentially as they would be supplied in the clinical setting. Times of diagnosis were compared using a two-tailed Wilcoxon paired ranked sum test.

\section{Results}

A definite deterioration in renal function (rejection categories 1 or 6 ) was identified by the clinician on 32 occasions, on 28 of which this was due to rejection (tables I and II). Twenty nine of these episodes were detected by the statistical analysis using only plasma creatinine results, and 25 when using only plasma urea results (including two not detected using plasma creatinine). On a single occasion the computer method failed to detect a deterioration in renal function considered clinically important, both at the time and in retrospect. In this the only false negative incident clinical diagnosis was based on two rising creatinine values, one morning and one evening measurement, the latter of which was not able to be used in the statistical technique at that phase of its development as reported here. The high evening result was followed by a fall in creatinine and the deterioration in function failed to reach significance when the statistical analysis was applied.

When creatinine data were analysed without applying any weight correction $^{11}$ for acute changes in hydration the rate of detection fell from 29 to 26 out of the 32 episodes, reflecting the importance of this adjustment.

Using weight corrected plasma creatinine concentrations the computer identified rejection a median of one day earlier $(p<0.02)$ than treatment was actually started by the transplant team (table II). The team in Nottingham at that time used hand drawn graphs of the weight corrected reciprocal of plasma creatinine and were, therefore, better able to identify rejection early than those groups who do not use these methods. Computer identification was also significantly earlier $(p<0.05)$ than the retrospective clinical diagnosis of rejection (made by MSK), diagnosing rejection seven days earlier in one instance. Using the statistical method rejection was diagnosed consistently earlier using weight corrected creatinine rather than urea concentrations $(p<0.01)$, and there was no significant difference between the time of a statistical diagnosis based on plasma urea and the clinical diagnosis of rejection.

The difficulty of defining a disturbance of renal function is illus-

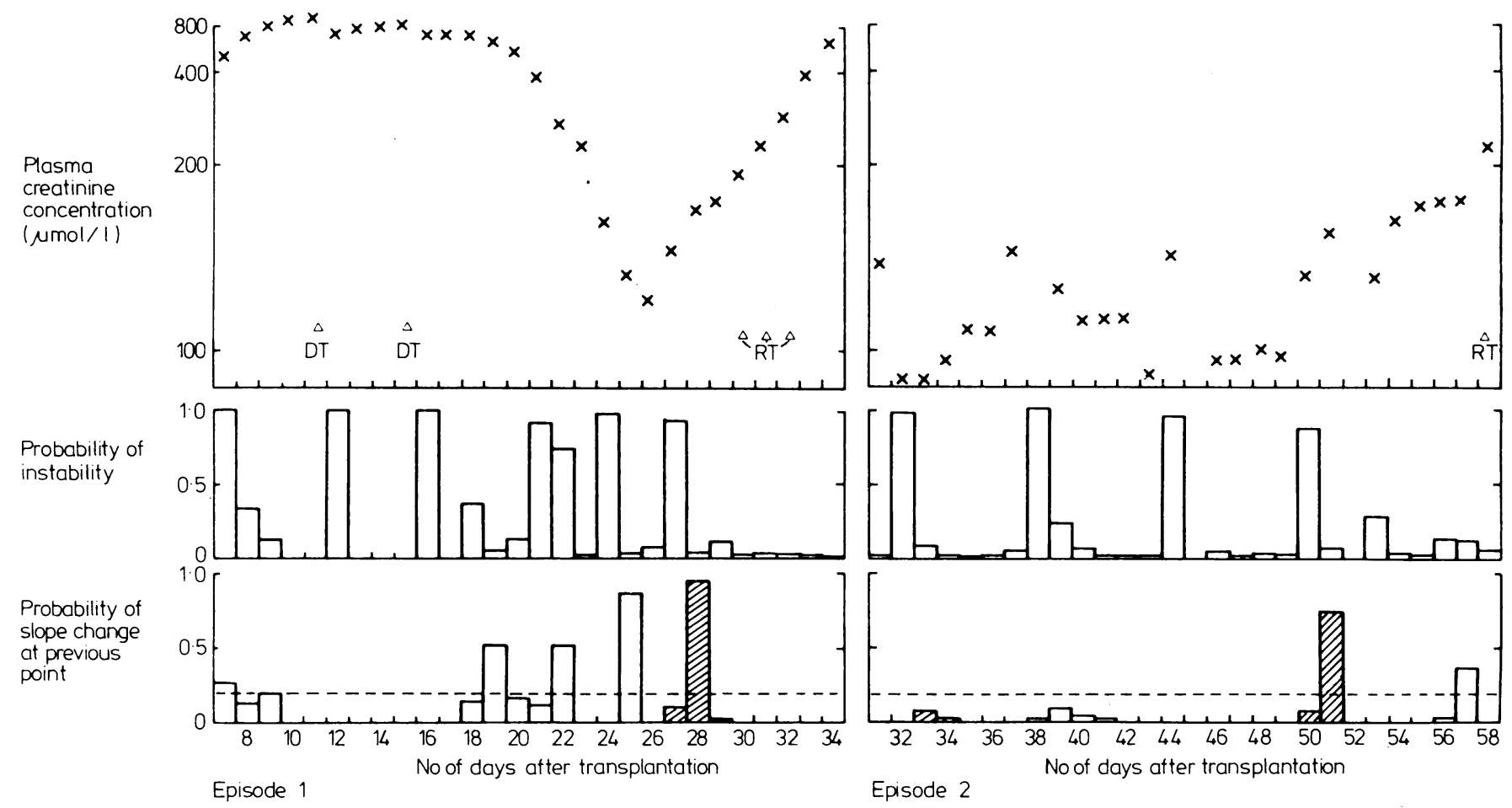

FIG 3-Kalman filter analysis of two rejection episodes (clinical rejection category 6). Upper graph shows weight-corrected plasma creatinine concentration on an inverted reciprocal scale plotted against time (days after transplantation). Middle graph shows probability that there is some form of instability in system at time $\mathrm{t}$. Lower graph shows probability that a slope change occurred at $\mathrm{t}-1$, given value at time $\mathrm{t}$ : hatched bars indicate a positive slope change-that is, a deterioration in function-values exceeding the cutoff graticule being defined as significant. DT $=$ Dialysis, RT $=$ antirejection treatment.

Conversion: SI to traditional units-creatinine-1 $\mu \mathrm{mol} / 1 \sim 0 \cdot 01 \mathrm{mg} / 100 \mathrm{ml}$.

Episode 1 Low noise series. Instability detected on day 27 is confirmed as a positive slope change on day 28 , indicating a significant deterioration in renal function. Dialysis on days 11 and 15 resulted in level changes that are detected as instability but are not confirmed as slope changes.

Episode 2 High noise series. System adjusts so that it is less sensitive to transient disturbances (middle graph) in a "noisy" series, but a deterioration in function is detected on day 51 and was treated as rejection seven days later. 
trated by the fact that rejection could be suspected clinically on 97 days (categories $1,2,3,4$, and 6), but of these only 28 (category 6) were confirmed by the further investigation (repeat measurement of creatinine, immunology, renal scan, or biopsy). The clinician did not suspect rejection (category 0 ) on 11 days on which the computer did detect a significant deterioration in function using weight corrected creatinine data. If statistical analysis had been used to complement clinical review for these patients additional investigations would have been performed on 108, rather than 97 , occasions out of 1356 patient days considered. There remains uncertainty as to whether these 11 episodes represent a biological event of clinical importance. Of the 65 episodes considered by MSK to be a possible deterioration in function (categories 2, 3, and 4) the computer identified 25. (The uncertainty of the clinical diagnosis of rejection was related to the proportion of clinical events associated with a computer diagnosis: definitely no deterioration, $11 / 1259=0.01$; possible deterioration, $25 / 65=0 \cdot 38$; definite deterioration, $29 / 32=0 \cdot 91$.)

\section{Discussion}

Graphical presentation of results, after appropriate transformations when necessary to, for example, the log or reciprocal, offer a useful way of considering the long sequences of data collected from patients. ${ }^{13-16}$ Computers are already being used by some renal units, including our own, to store data and present them instantly as graphs. ${ }^{17} 18$ These units have the facility to make mathematical transformations, calculate corrections for acute changes in volume of distribution, and perform statistical analyses. We investigated the ability of the computer to perform statistical analyses and demonstrated that statistical programs may define an event as well as or better than an experienced clinician working in retrospect.

If, for purposes of comparison, rejection episodes were defined objectively as the time that the computer identifies

TABLE I-No of times a deterioration in renal function was diagnosed by computer and by clinician for each of six clinical categories. These categories may be grouped into those where there was definitely no deterioration in renal function $(0$ and 5$)$ and those in which a definite deterioration in function occurred (1 and 6). Sensitivity and specificity of weight corrected creatinine and urea and of uncorrected creatinine were calculated from these two groups. In addition, there were 65 days when there was uncertainty whether a deterioration in function had occurred $(2,3$, and 4$)$

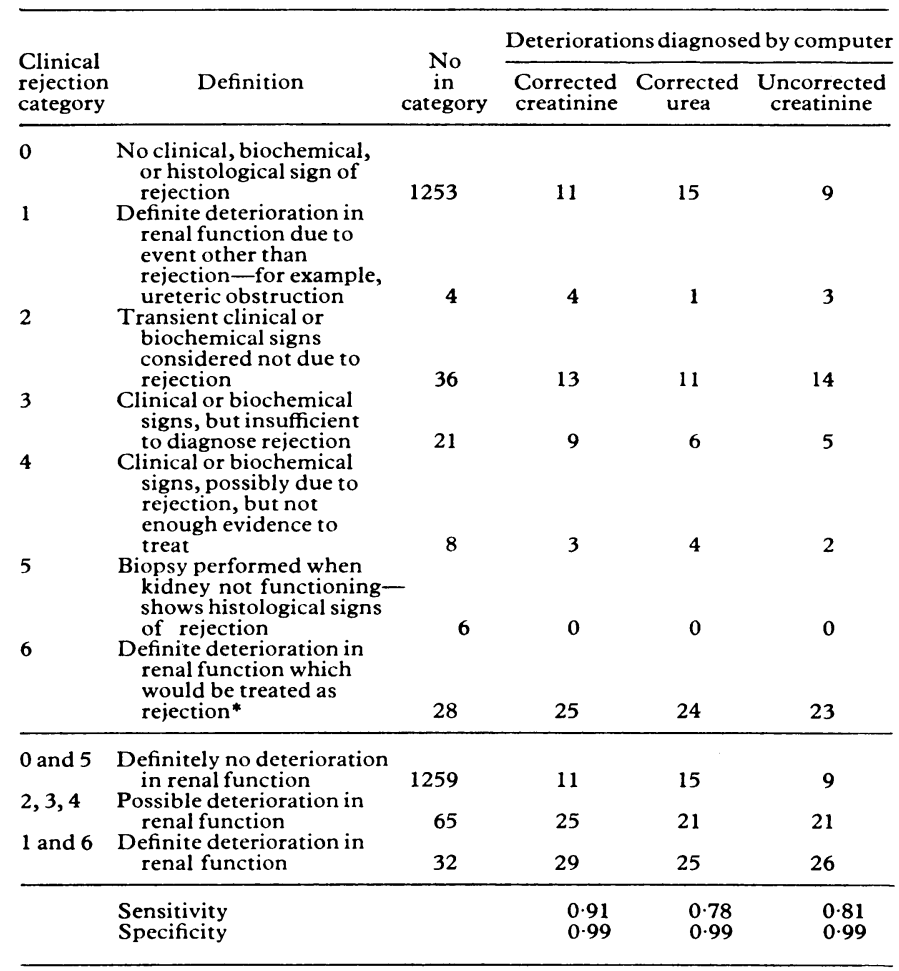

*Positive findings in all 14 biopsies undertaken.
TABLE II-Time of diagnosis of allograft rejection (days after transplantation) definitions of timing are given in methods section

\begin{tabular}{|c|c|c|c|c|}
\hline \multirow{2}{*}{$\begin{array}{l}\text { Episodes of } \\
\text { rejection }\end{array}$} & \multicolumn{2}{|c|}{ Clinical diagnosis } & \multicolumn{2}{|c|}{ Computer diagnosis } \\
\hline & Prospective & Retrospective & Creatinine & Urea \\
\hline \multicolumn{5}{|l|}{ Category $1^{*}$ : } \\
\hline 1 & $8 \cdot 5$ & $9 \cdot 5$ & $10 \cdot 5$ & \\
\hline 2 & & $18 \cdot 5$ & $16 \cdot 5$ & \\
\hline 3 & & 28.5 & 28.5 & \\
\hline 4 & & $5 \cdot 5$ & $5 \cdot 5$ & $6 \cdot 5$ \\
\hline \multicolumn{5}{|l|}{ Category $6^{*}:$} \\
\hline 1 & $28 \cdot 5$ & $28 \cdot 5$ & $29 \cdot 5$ & $29 \cdot 5$ \\
\hline $\begin{array}{l}2 \\
3\end{array}$ & $45 \cdot 5$ & $44 \cdot 5$ & $43 \cdot 5$ & $45 \cdot 5$ \\
\hline $\begin{array}{l}3 \\
4\end{array}$ & $24 \cdot 5$ & $\begin{array}{l}12.3 \\
24.5\end{array}$ & $22 \cdot 5$ & 23.5 \\
\hline 5 & $29 \cdot 5$ & 28.5 & $29 \cdot 5$ & \\
\hline 6 & $59 \cdot 5$ & $59 \cdot 5$ & $59 \cdot 5$ & \\
\hline 7 & $25 \cdot 5$ & $25 \cdot 5$ & $22 \cdot 5$ & 22.5 \\
\hline 8 & 20.5 & $19 \cdot 5$ & 16.5 & 17.5 \\
\hline 9 & $23 \cdot 5$ & $21 \cdot 0$ & 16.5 & $18 \cdot 5$ \\
\hline 10 & 43.5 & 41.5 & 39.5 & 42.5 \\
\hline 11 & 7.5 & 8.5 & $8 \cdot 5$ & 8.5 \\
\hline 12 & $4 \cdot 5$ & $5 \cdot 5$ & $5 \cdot 5$ & $5 \cdot 5$ \\
\hline 13 & $19 \cdot 5$ & $19 \cdot 5$ & 20.5 & 22.5 \\
\hline 14 & $42 \cdot 5$ & 41.5 & $41 \cdot 5$ & $43 \cdot 5$ \\
\hline 15 & 6.5 & 5.5 & 6.5 & $5 \cdot 5$ \\
\hline 16 & $67 \cdot 5$ & $58 \cdot 5$ & $51 \cdot 5$ & \\
\hline 17 & $5 \cdot 5$ & $6 \cdot 0$ & 6.5 & $5 \cdot 5$ \\
\hline 18 & $19 \cdot 5$ & 19.5 & 17.5 & $19 \cdot 5$ \\
\hline 19 & 31.5 & $29 \cdot 5$ & 29.5 & $30 \cdot 5$ \\
\hline 20 & $6 \cdot 5$ & 6.5 & 5.5 & 5.5 \\
\hline 21 & $23 \cdot 5$ & $23 \cdot 5$ & $23 \cdot 5$ & $23 \cdot 5$ \\
\hline 22 & $44 \cdot 5$ & 43.5 & $43 \cdot 5$ & 40.5 \\
\hline 23 & 17.5 & $16 \cdot 5$ & 16.5 & 17.5 \\
\hline 24 & $10 \cdot 5$ & $11 \cdot 0$ & $11 \cdot 5$ & 11.5 \\
\hline 25 & & 42.5 & & $39 \cdot 5$ \\
\hline 26 & 6.5 & 7.5 & $7 \cdot 5$ & $7 \cdot 5$ \\
\hline $\begin{array}{l}27 \\
28\end{array}$ & $\begin{array}{l}27 \cdot 5 \\
35 \cdot 5\end{array}$ & $\begin{array}{l}27 \cdot 5 \\
33 \cdot 5\end{array}$ & 33.5 & $\begin{array}{l}28.5 \\
33.5\end{array}$ \\
\hline & & & & \\
\hline
\end{tabular}

*See table I for definitions.

an appreciable deterioration in function, the incidence of acute episodes of renal failure in centres using different regimens could be quantified and compared. The objective definition of the time of computer diagnosis of rejection may also be used to determine whether changes in other variables-for example, urea, $\beta 2$ microglobulin, urinary solute excretion-precede or follow changes in plasma creatinine trend. In this study the change in creatinine trend associated with rejection was shown to precede the change in urea by an average of one day $(p<0.01)$.

Our results show that, at the current state of development, the method has the potential to analyse incoming results online and so help inexperienced clinicians to identify potentially important changes. To establish its usefulness as a prospective method for detecting dysfunction a prospective study is required to investigate the patient when either the computer or the clinical team identify a deterioration in function. With the recent trend towards computerisation of renal records ${ }^{18}$ the method is ready for incorporation into the day to day care of patients. We studied four patients prospectively over a total of 130 patient days. A definite deterioration in renal function (categories 1 or 6 ) was identified by the clinicians on six occasions, all of which were due to rejection. The computer detected all six episodes, a median of one day earlier than treatment was initiated by the transplant team. A deterioration in function was suspected clinically (categories 2, 3, and 4) on a further five occasions, of which three were signalled by the computer. Thus, when applied to detect dysfunction in this small sample of patients the method had a sensitivity of 1.00 and a selectivity of 1.00 -that is, no false positives or negatives.

Future developments of the statistical technique should allow dependent variables such as creatinine, urea, and $\beta 2$ microglobulin, to be considered concurrently in a single analysis. A false negative result for one variable may be offset by a true positive result for another variable, thus improving the sensitivity in contrast to when the same variables are considered independently. The Kalman filter technique has also been adapted to analyse urinary data from patients who have had transplants. Three eight hourly specimens were collected each day with the results considered as three simultaneous series, the computer analysing more information than could reasonably be considered prospectively by any clinician alone. Methods such as these could make a valuable contribution to the care of patients 
with other illnesses necessitating intensive monitoring of numerical data, ${ }^{9}$ and to the evaluation of series of data collected in the course of research.

We thank Professor Roger Blamey, Dr Richard Burden, and the many other colleagues who helped in the care of these patients and in their investigations. The study was supported by grants from the Medical Research Council and the Nottingham and Nottinghamshire Kidney Fund.

\section{References}

1 Cosimi AB, Colvin RB, Burton RC, et al. Monoclonal antibodies for immunological monitoring and treatment in recipients of renal allografts. $N$ Engl f Med $1981 ; 305: 308-14$.

${ }^{2}$ Foegh ML, Winchester JF, Zmudha, et al. Urine $\mathrm{i}-\mathrm{TXB}_{2}$ in renal allograft rejection. Lancet 1981 ;ii:431-4.

${ }^{3}$ Knapp MS, Cove-Smith JR, Dugdale R, Mackenzie N, Pownall R. Possible effect of time on renal allograft rejection. Br Med f 1979;i:75-7.

${ }^{4}$ Harrison PJ, Stevens C. Bayesian forecasting. Fournal of the Royal Statistical Society. Series B. 1976;38:205-47.

${ }^{5}$ Freedman AM, Duneman OP, Peckham G, Trattner A. Automatic recognition of significant events in the vital signs of neonatal infants. Comput Biomed Res 1978;12:141-8.

${ }^{6}$ Wilson DW, Griffiths K, Kemp KW, Nix ABJ, Rowlands RJ. Internal quality control of radioimmunoassays: monitoring of error. $\mathcal{F}$ Endocrinol $1979 ; 80: 365-72$.

${ }^{7}$ Chik L, Sokol RJ, Rosen MG, Pillay SK, Jarrell SE. Trend analysis of intrapartum monitoring data: a basis for a computerised fetal monitor. Clin Obstet Gynaecol 1979;22:665-79.

${ }^{8}$ Royston JP, Abrams RM. An objective method for detecting the shift in basal body temperature in women. Biometrics $1980 ; 36: 217-24$.

${ }^{9}$ Stoodley KDC, Mirnia M. The automatic detection of transients, step changes and slope changes in the monitoring of medical series. Statistician $1979 ; 28: 163-70$.

10 West M, Smith AFM. Monitoring renal transplants: an application of the multi-process Kalman Filter. Biometrics (in press).

${ }^{11}$ Knapp MS, Blamey RW, Cove-Smith JR, Heath M. Monitoring the function of renal transplants. Lancet 1977; ;i:1183.

12 Van der Helm HJ, Hische EAH. Application of Bayes's theorem to results of quantitative clinical chemical determinations. Clin Chem 1979;25: 985-8.

${ }^{13}$ Knapp MS, Smith AFM, Trimble I, Pownall R, Gordon K. Mathematical and statistical aids for evaluating sequences of data collected from renal patients. Kidney Int (in press).

14 Jelliffe RW, Jelliffe SM. A computer program for estimation of creatinine clearance from unstable creatinine levels, age, sex and weight. Mathematical Biosciences 1972;14:17-24.

15 Mitch WE, Walser M, Buffington GA, Lemann J. A simple method of estimating progression of chronic renal failure. Lancet 1976;ii:1326-8.

16 Rutherford WE, Blondin J, Miller JP, Greennalt AS, Vavra JD. Chronic progressive renal disease: rate of change of serum creatinine concentration. Kidney Int 1977;11:62-70.

17 Gordon M, de Wardener HE, Venn JC, Webb JT, Adams H. The interactive graphic database microcomputer for clinical use in data intensive therapies. Proc Eur Dial Transplant Assoc 1981;18:690.

18 Anonymous. Kidneys and computers [Editorial]. Br Med $\mathcal{F} 1980$;281: 1302-3.

(Accepted 17 December 1982)

\title{
Successful treatment of herpes labialis with topical acyclovir
}

\author{
A PAUL FIDDIAN, JANE M YEO, RONALD STUBBINGS, DONALD DEAN
}

\begin{abstract}
A double blind, placebo controlled trial of $5 \%$ acyclovir cream, applied topically five times a day for five days, was carried out in 49 patients with recurrent herpes labialis. These patients had a total of 74 episodes, 34 of which were treated with the $5 \%$ acyclovir cream and 40 with matching placebo. First episodes and all episodes treated with acyclovir cream had significantly shorter times to formation of ulcer or crust and to complete healing ( $p<0.05$ for all variables). The duration of all symptoms and proportion of patients developing itching was also reduced by acyclovir cream in first episodes, though the difference was not significant. When the patient started treatment early in the course of a first episode acyclovir cream significantly reduced the per-
\end{abstract}

Department of Clinical Immunology and Chemotherapy, Wellcome Research Laboratories, Beckenham, Kent BR3 3BS

A PAUL FIDDIAN, MB, research physician

JANE M YEO, PHD, clinical research assistant

Medical Services, British Petroleum Company Limited, Britannic House, London

RONALD STUBBINGS, $\mathrm{MB}$, medical officer

DONALD DEAN, MB, head of medical services

Correspondence and requests for reprints to: Dr A Paul Fiddian. centage of lesions progressing beyond the papular stage $(\mathbf{p}<\mathbf{0 . 0 5})$.

Acyclovir cream is well tolerated and effective for the treatment of recurrent herpes labialis.

\section{Introduction}

Recurrent herpes simplex infections around the mouth (herpes labialis, cold sores, fever blisters) occur in up to one fifth of young adults, ${ }^{2}$ but no treatment has hitherto been shown in double blind, placebo controlled trials to have an appreciable clinical benefit. The discovery of acyclovir (Zovirax, Wellcome), an antiviral drug with highly specific activity against herpes simplex virus types 1 and $2,{ }^{3}$ offers the possibility of a safe and effective topical preparation for the treatment of recurrent herpes simplex virus infections. Oral acyclovir has been shown to be effective in the treatment of recurrent genital herpes, and topical acyclovir in an ointment base (polyethylene glycol) shortens the duration of virus shedding in patients with recurrent genital herpes ${ }^{5}$ and herpes labialis, ${ }^{6}$ without producing a demonstrable clinical benefit. The limited effect of local antiviral treatment in recurrent herpes simplex virus infections may be due to poor skin penetration of the drug, and on this account it has been suggested that acyclovir be applied using dimethyl sulphoxide as the vehicle. ${ }^{6} 7$ An alternative formulation using propylene glycol as solvent has been found to be superior to acyclovir ointment for the treatment of experimental cutaneous herpes simplex virus infections in animals. ${ }^{8}$ 